\title{
A Research Effort to Categorize Social Posts Applying Two Phase Natural Language Processing Methodology
}

\author{
Geetanjali R. Salunke ${ }^{1}$, S. S. Patil ${ }^{1}$, Debnath Bhattacharyya ${ }^{2}$ and Hye-Jin Kim ${ }^{3}$ \\ ${ }^{1}$ Bharati Vidyapeeth University College of Engineering, Pune \\ ${ }^{2}$ Department of Computer Science and Engineering, \\ Vignan's Institute of Information Technology, \\ Visakhapatnam-530049, India \\ ${ }^{3}$ Sungshin Women's University, 2, Bomun-ro 34da-gil, \\ Seongbuk-gu, Seoul, Korea \\ nalini.r.salunke@gmail.com,sspatil@bvucoep.edu.in,debnathb@gmail.com, \\ hyejinaa@daum.net \\ (Corresponding Author)
}

\begin{abstract}
Today with Advancement in network and Web Technology ratio of internet users is counting up. In current scenario, users are so much comfortable to Social Networks. Different OSN (Online Social Network) are used to connect with each other. Facebook is an emerged as vital source of communication. But security has been overlooked. Most of the facebook user spread and sent various type of content such as post messages, images, URLs, News about distinct topics, But major problem with facebook and other OSN a large amount of adult content are spread very rapidly through internet. Adult content are in the form of messages, URLs (Links containing adult web pages), images. Through internet people can easily spread adult content on facebook and OSN. There is need to provide the security to online social networks from adult content spread through web. We are proposing an effective solution for facebook content classification into adult and non adult. In this paper we are proposing an application which is used to classify facebook content into adult and non adult content at text and URLs level. To classify the facebook content into adult and non adult content we use natural language processing and machine learning techniques. We then categorize the classified facebook content into different topics such as business and industrial, law, government and politics, news and others. In addition, we enlarge our system for facebook data classification based on facebook post and comment posted on own wall and graphically represent overall classification of adult and non adult posts and categorization of user post.
\end{abstract}

Keywords: Natural Language Processing, Adult content, Facebook Post, URLs, Machine Learning

\section{Introduction}

In the past, Internet was not in a lot use. Nowadays, use of internet is increasing extremely, speedily, concurrently users who are exciting to do their highest work on internet. Today Online Social Networks (OSNs) are very trendy platform. The major favorite OSNs are Twitter, Facebook, LinkedIn etc. With the increasing popularity of these OSNs, the assaults on OSNs are also increasing. Using different OSNs people share their opinion, feeling and thoughts. There are large numbers of active users on OSNs. Online social networking sites are the major ways for OSNs users to stay in touch with people be in touch with their friends through online. Facebook is one of the most top popular Social networking sites mostly viewed of the Internet [10]. The majority of these 
sites offers platform for users to sharing emotions, opinion and post messages on facebook about different topics. Internet user shares his opinion and emotions about different events. Facebook is most popular platform for sharing information with friends. It also allow user to communicate with each other, sharing opinion, emotions and updating status of own.

Online social networking sites share a various type of technical features that permit the persons to make a public profile and can maintain some contents of profile personal noticeable only to selected friends and clear the users list that they share connection and can see their connections list inside the system. In facebook there is a visible profile of users which contains the list of user friends those are the user of the facebook site. On online social network sites a profile is generated from getting the answers of several common questions, such as place, age, interests etc. Facebook allows user to upload images or pictures and can also add multimedia content to change the view of the profile. Facebook allows user to develop their profile by giving facility to add applications. Facebook allows the users to post their status and search for other peoples with similar interests and share with friends. User profiles contain an area to comments to friends and other users. Facebook have controls that permits users to decide who can view their profile and get in touch with them, include them to the list of contacts, list of friends and all these will be done to defend user privacy, but up till now online social networks give small support to users and provide the ability to control the messages posted on their own private space to stay away from unwanted content to be displayed. Generally profiles of users are not private but we can see the page of user if user having created connection with the trusted user. If facebook user wants to become friend with a new user, then he sends a friend request to that user which is known to him very well. When that user recognize him then he accept his friend request in this way the relation between those users are established [10]. Inside facebook the large spread of adult content is an upcoming problem faced by facebook user and other OSNs. The main reason of spreading adult content on facebook is facebook user sent and shares tweets, messages, URLs with his facebook friend, but user friend sent and share message with his another facebook friend. Content sharing between them may be normal content or adult content such as adult messages, adult website (URLs), but the user friend do not interested to look such adult content on his facebook wall. In this paper we are proposing an effective solution of this problem faced by facebook user. We classify the facebook data into adult and non adult content at text and URLs level. We are using natural language processing for the classification of facebook data. Our main aim is to classify the facebook data for increasing the facebook user experience and providing more security from unwanted data. To classify the facebook data from facebook user wall we will going to use machine learning based classification and NLP (natural language processing). In facebook, classification of adult content can also be used for susceptible issues. Since the actuality that in facebook there is the option of posting or commenting other posts on specific public or private area, called in common walls. But, no content-based preferences are supported and therefore it is impossible to avoid undesired messages, such as political or bad ones. So data classification can be used to give users ability to automatically classify the post into adult and non adult written on their own facebook walls. Facebook user shares messages, post tweets, comments about any subject or topics with their friends. In this paper we initiate our method and make a system which includes three tasks collectively.

Task 1: In case of detecting adult content, we prepare model for Detecting adult post on the facebook for better classification of facebook data. Here, our most important aim is to focus on adult post and adult URLs that can appear in facebook user posts, comments, messages, links posted by user. It determines the adult content which presents inside posts such as adult words. It also determines the adult URLs which posted inside posts such as 
blacklisted URLs there are various type of adult URLs. In this way our system classifies the facebook post into adult and non adult content at text and URLs level.

Task 2: In second task, we prepare system for post summarization. Here, we recognize the interesting or important part of the posts and URLs presents in the users post and rate the posts by scoring it based on different posts and URLs features appearing within given post. In addition, we enlarge this system to signify this classification, categorization wise in graphical representation design which is stated in the third task.

Task 3: Graphical representation for facebook content is based on overall posts of that user by counting the score of posts and URLs according to adult content (Post and URL), non adult content (Post and URLs) classes. The essential idea behind graphical demonstration is to illustrate how many how many content are adult and non adult content (both at text and URL level). It also represents the category of post with score of categories.

The remaining part of this paper is organized as follows. In Section 2 we have cover survey about related work. Also we initiate different techniques proposed by different authors for adult content detection and classification. In Section 3 we initiate and signify our proposed system and flow of our proposed work. Section 4 presents experimental results of our proposed methodology. In Section 5, we conclude our paper with future work.

\section{Background and Motivation}

Author [1] proposed a new solution to efficiently classify Twitter accounts that include contain adult content. Author identifies adult account express finding technique on Twitter by using relation between Twitter accounts and persons in tweets. They presented classifier (ISC), which based on graph classification method opposed to the various relations. They concentrate on detecting adult content in account phase by considering three reasons first imperfect information communicated by tweet so it is not easy to detect a adult tweets on. They proposed method to identify the adult accounts on Twitter, using adult detection techniques such as URL blacklisting, text and image based adult content recognition techniques [12-16]. Adult image detection is not possible for twitter because of the high calculation cost and small accuracy. Over the previous year various techniques have been proposed for detection of adult web content. It controls various kinds of information such as textual content, picture, and URLs. Author proposed method to sort web pages containing bad words using NLP [15]. For text classification large training dataset are required, But current studies [17-19] specify that these techniques are responsive to surrounding sound, explanation inconsistency and picture superiority, so it do not execute well for images detained by using low feature web cameras prepared on cell phone procedures. Additionally, there are some limitations to detect the adult image because of vast number of images; videos are posted daily on OSN. URL blacklisting technique is used in data sorting software [20].

\subsection{Facebook Social Network}

Facebook is very popular OSN. There are number of active users on facebook. The status of facebook related to its features that create social communications sharing information which are more interactive. Facebook users interact with each other by sending messages and URLs on their wall. On facebook, user can give comments on posts [11].

Facebook has its own protected system [5] to defend facebook users from unnecessary malicious content. Researchers construct and organize a logical, more powerful and usually long instantaneous system to protect facebook users and public graph. In this difficult system originator used an exhaustive group of components and method to distinguish between justifiable procedures and spam. They used groups of components 
such as classifiers for classification. Author [6] proposed a primary study to measure and characterizes spam promotion begins using facebook accounts. Author [7] offered an online spam sorting system that might be organized as a part of the OSN platform to check messages created by user in real time. They focused on recreating spam post into promotions for sorting before observing every post separately.

\subsection{Detection of URLs}

Author [8-9] proposed method of URL analysis. The URLs are extracted from tweets are reduced ones and then it classified using HttpURL connection group use.

\section{URLs are analyzed by using following steps:}

\subsubsection{By Comparing with the Dataset}

Comparing posted URLs with Blacklisted URLs dataset. If posted URLs present in that dataset then that URLs are adult content URLs [10].

\subsubsection{Comparing with a Group of Previously Recognized Terms Inside URL}

Adult URLs are identified by using adult words inside the URLs. Adult URLs are recognized through different group of adult words inside URLs. Author [10] used collection of 33 adult words inside URLs to identify adult URLs.

In text classification author [4] proposed a text classifier based on short-text classification and analysis. In addition, the package is designed so that users can suitably make extensions. LibShortText is very helpful short-text classification and analysis. For short texts, they follow the bag-of-word model to generate features. In this library users can apply method to pre-processing short texts by tokenization, stemming and stop-word removal. It allows users to select between unigram and bigram features. In libshorttext classifier author proposed user can choose feature representation (binary, word count, Term Frequency or Term frequency-inverse document frequency (TF-IDF). The main motivation behind this work is the classification and detection of facebook content into adult and non adult classes at text and URLs level. Second motivation is to categorize the facebook data into various topics to increase the user performance.

\section{Methodology}

\subsection{Training Set for Classification}

In classification we have create one training set which contain the adult words which will be appearing in the users post. In URL classification we have use the dataset of blacklisted URL which is available on web. Execution of our proposed system is completed by using following techniques and methods.

Natural Language Processing: In this technique machine learns the natural language like English language and then executes every task which a human can do. In [11], authors have used concepts for NLP:

\section{Tokenization:}

Tokenization is the process that divided sentences in the post into stream of tokens such as words. It gets one by one sentence in the post to tokenize each word.

2. Remove Stop Words: In this task stop words are removes from post in the text sentences.

\section{Stemming:}

After removing stop words it removes suffixes from post. Stop words are eliminated first then stemming algorithm is implemented. If any word in post present into list of adult words then that will be considered as adult post at text level. Firstly, post having vulgar 
and obscene language, ambiguous comments are eliminated. Then, preprocessing of post will be done. Thus, when any such property declared above is found the post will be defined as adult post.

\subsection{POS Tagging}

Part-Of-Speech tagging (POS) is necessary to recognize Verb, Noun and Noun Phrases in post in text form. As we know that grammatical texts are classified by assuming eight different POS. So our systems apply this tagging to execute some task. Part-of Speech parses each word of given post (text) and identifies that word according to its category (whether the word is a noun, verb adjective) etc.

\subsection{Data Collection}

For facebook data classification into adult and non adult, we extract Facebook post (text and URL) from facebook social network. We call to facebook API (developers.com) [3] for extracting post from facebook account. For each event, category and topics facebook post may be different; some post words may have single meaning for each category. Facebook user can give their response of post in terms of likes, reply, comment and shares for a particular post. Here, we focus on extracting two types of features of post such as unigram and bigram. Unigram features are simply BOW features and signify by using BOW model to form the text into many set of words by removing noisy characters and extra spaces in post which is in text form. Each two successive words of text in post are represented by using Bigram features. We execute this system via OpenNLP tool and their libraries. We also required some external resources such as facebook graph API.

\section{Algorithm}

\section{Algorithm: Two Phase NLP Processing}

\section{Phase 1: URL Process Algorithm}

\section{Input: URL 's}

\section{Process:}

1.URL vector <UV>

2.Blacklist URL vector $\langle\mathrm{BV}>$

3.cmp(UV,BV)

4.non_adult(UV)

Output: non adult(UV)

Phase 2: Text Categorization Algorithm

\section{Input: Text Post}

\section{Process:}

\section{Preprocess}

6. Sentences $(\mathrm{s}, \mathrm{c})$

7. Segmentation

8.non_adult(seg) 
Output: non adult(seg)

Categorized $(4,8)$

Graphevaluation();

\section{Proposed System Architecture}

We proposed a system for analysis, classification and categorization of facebook data by managing the problem of adult content detection by considering the posts on facebook. Here we proposed system for sentence level post classification to classify user's post into adult and non adult class labels. We first need to extract post from facebook. Using facebook API (developers.com) facebook posts are extracted for classification. For classification and categorization, we first required to identify whether the extracted posts are simple or complex sentences. We apply NLP task on posts (sentences). When the extracted post is simple sentence and states adult post/words/symbols, we train classifier to directly find content according to its classification classes i.e. adult content and non adult content class labels. Similarly, we train our system to compare extracted URLs with blacklisted URLs. In this way classification is done at text and URLs level. For categorization of classified posts we use training sets. Figure 1. represents the flow of our proposed system.

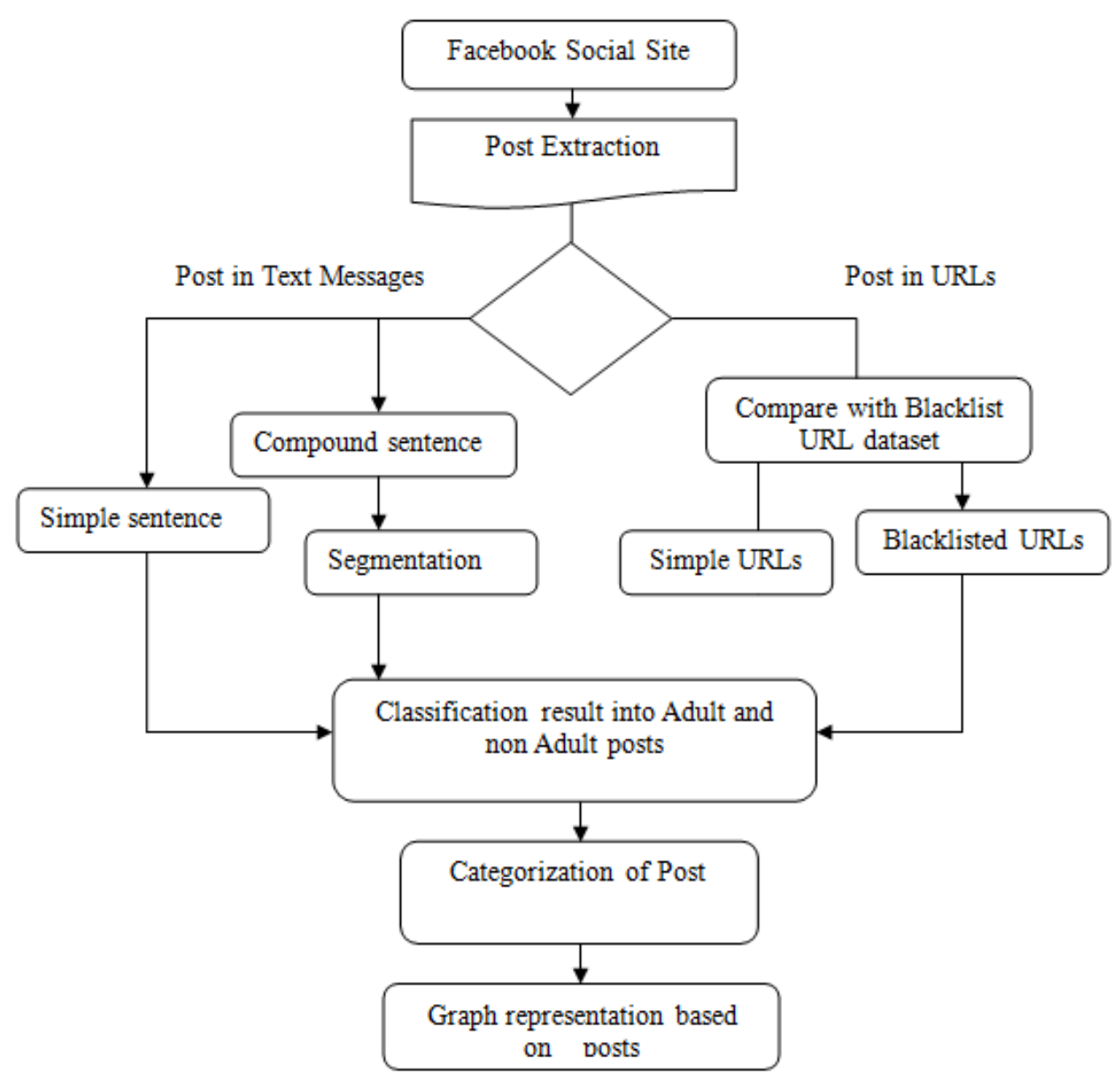

Figure 1. Proposed System Architecture 


\subsection{The Tasks of Our Proposed System are as Follows}

Task 1: In text level classification of post, here we first find out the out the features of post and opinion targets on which users have put their posts after that we apply NLP task and training set to classify users posts(text) into adult content and non adult content. In URL level classification, here we first download the group of Blacklisted URLs [2]. This dataset contains lots of URLs from distinct types. Here we have chose adult contents category. Our system compares extracted URLs from post with the dataset of blacklisted URLs. If the extracted URLs is present in the set of blacklisted URLs then that URLs are adult contents URLs. We also train system to calculate adult and non adult content score based on posts data.

Task 2: In this task, we categorize the classified data into different category as we have mentioned above. Here, we have used training set which contains words about related category. Our system compares the classified post with training set; if the category related words are found in the post (text form) then that post will be categorized into business, politics and others.

Task3: In third task, we graphically represent the score of adult and non adult content of users. We have also represents the category of classified post into different topics.

\section{Result Analysis}

Here we have represents experimental results for our proposed system; we apply this experiment on two dataset of post (text and URL) that we have mentioned above.

Figure 2: Login screen of facebook.

Description: Figure 2 Shows login screen of the facebook account.

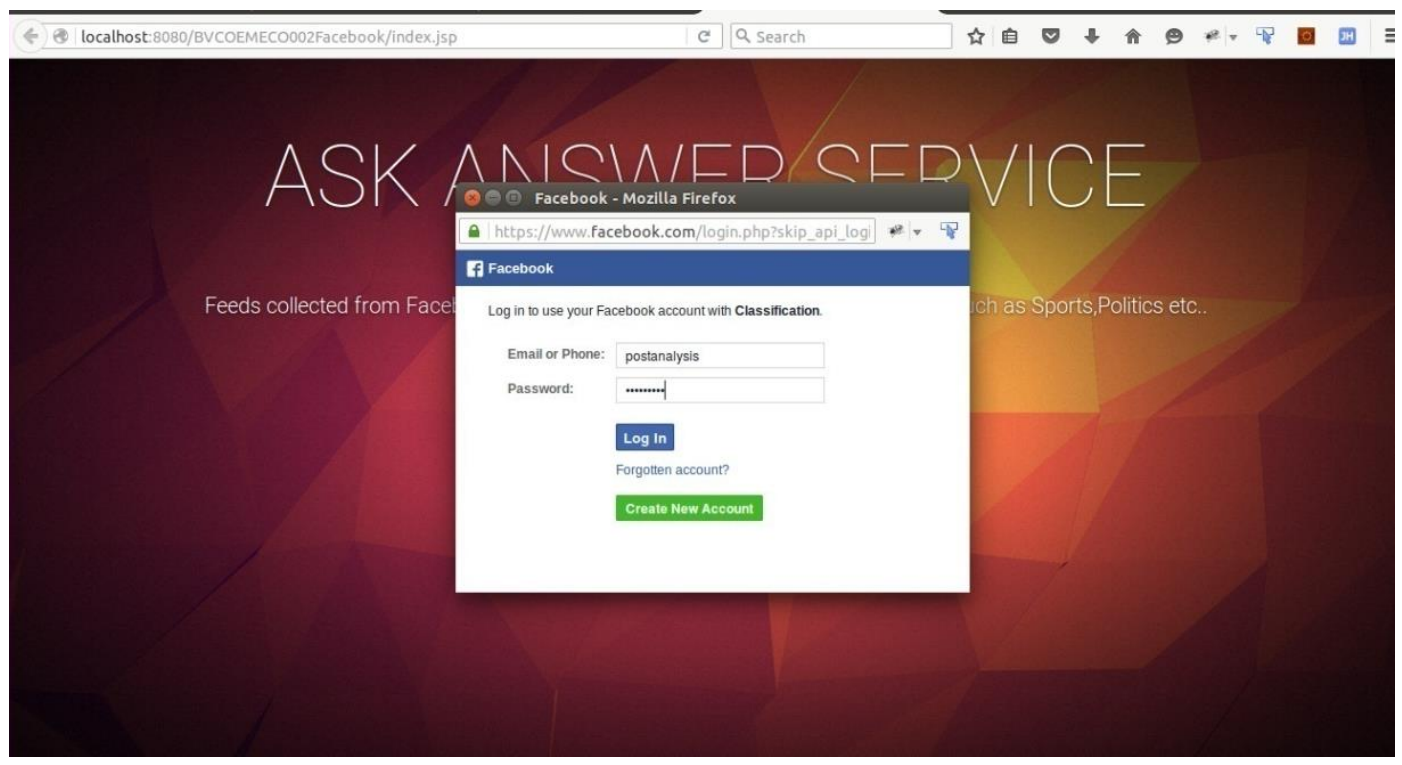

Figure 2. Login Window of Facebook 
Figure 3: Post on facebook.

Description: Figure 3 Shows post on facebook in sentence form

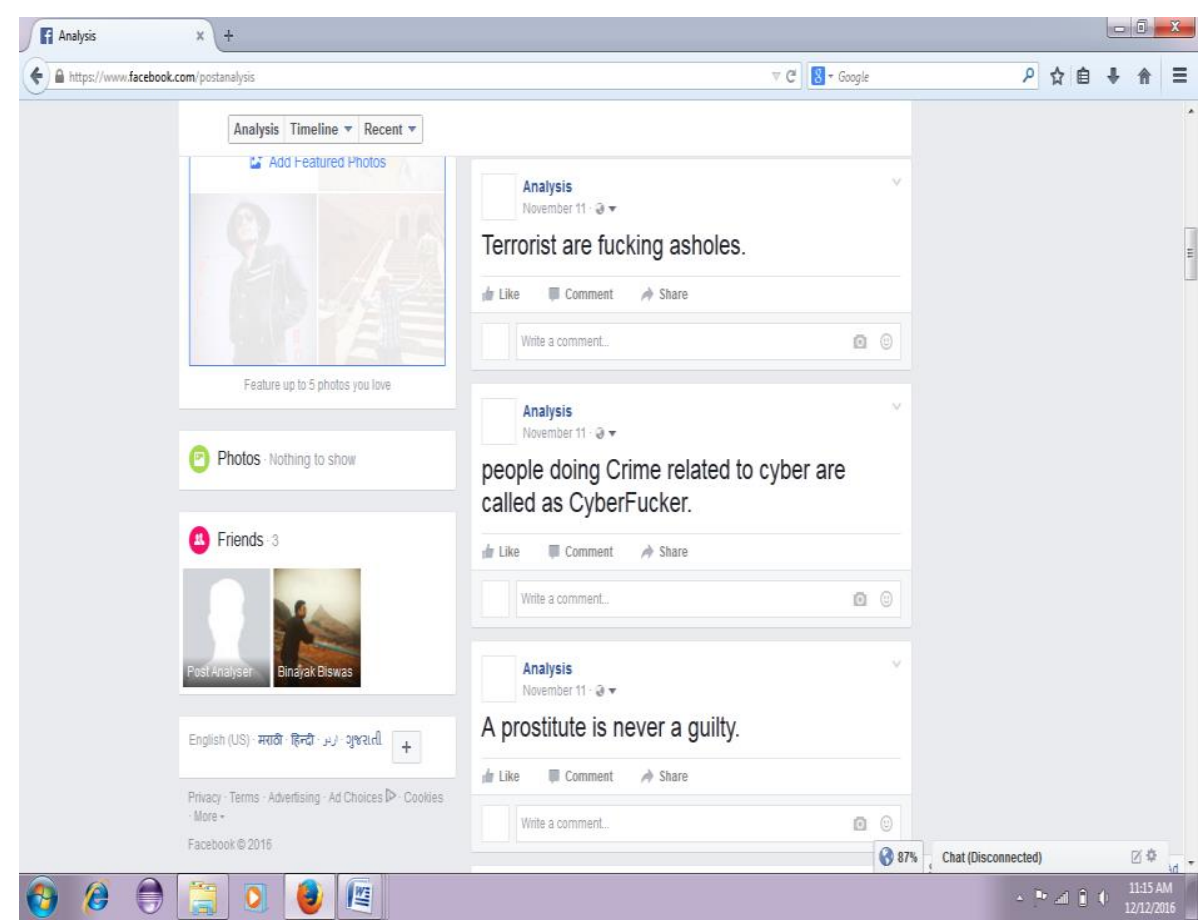

Figure 3. Post on Facebook

Figure 4: URLs posted on facebook.

Description: Figure 4 Shows URLs posted on facebook for classification.

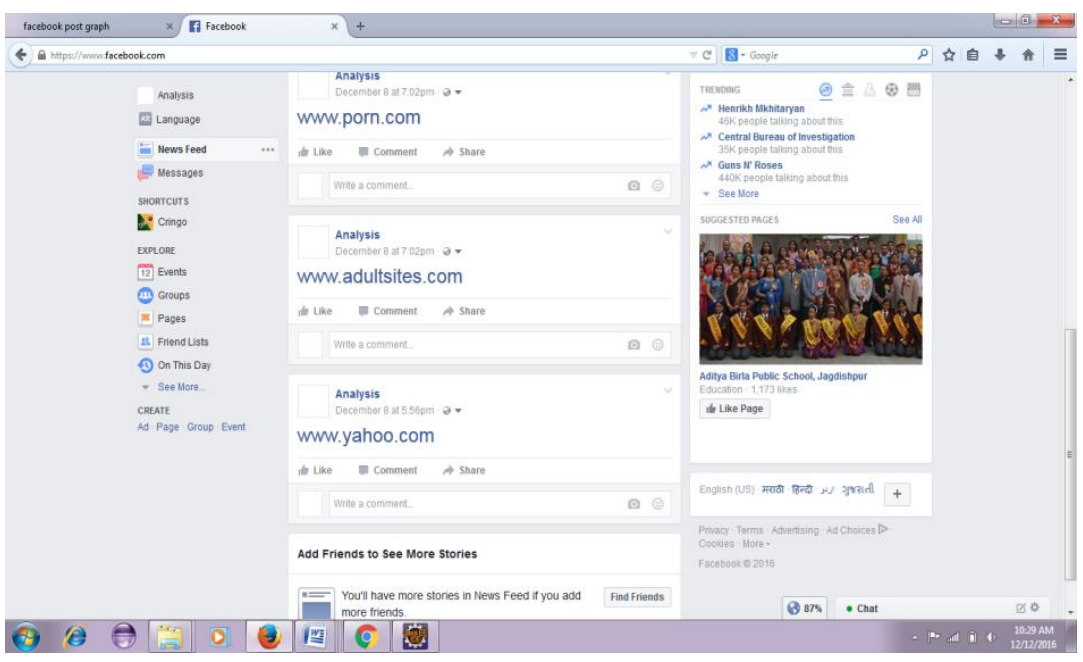

Figure 4. URLs Posted on Facebook 
Figure 5: classification and categorization result.

Description: Figure 5 Shows classification and categorization result with score.

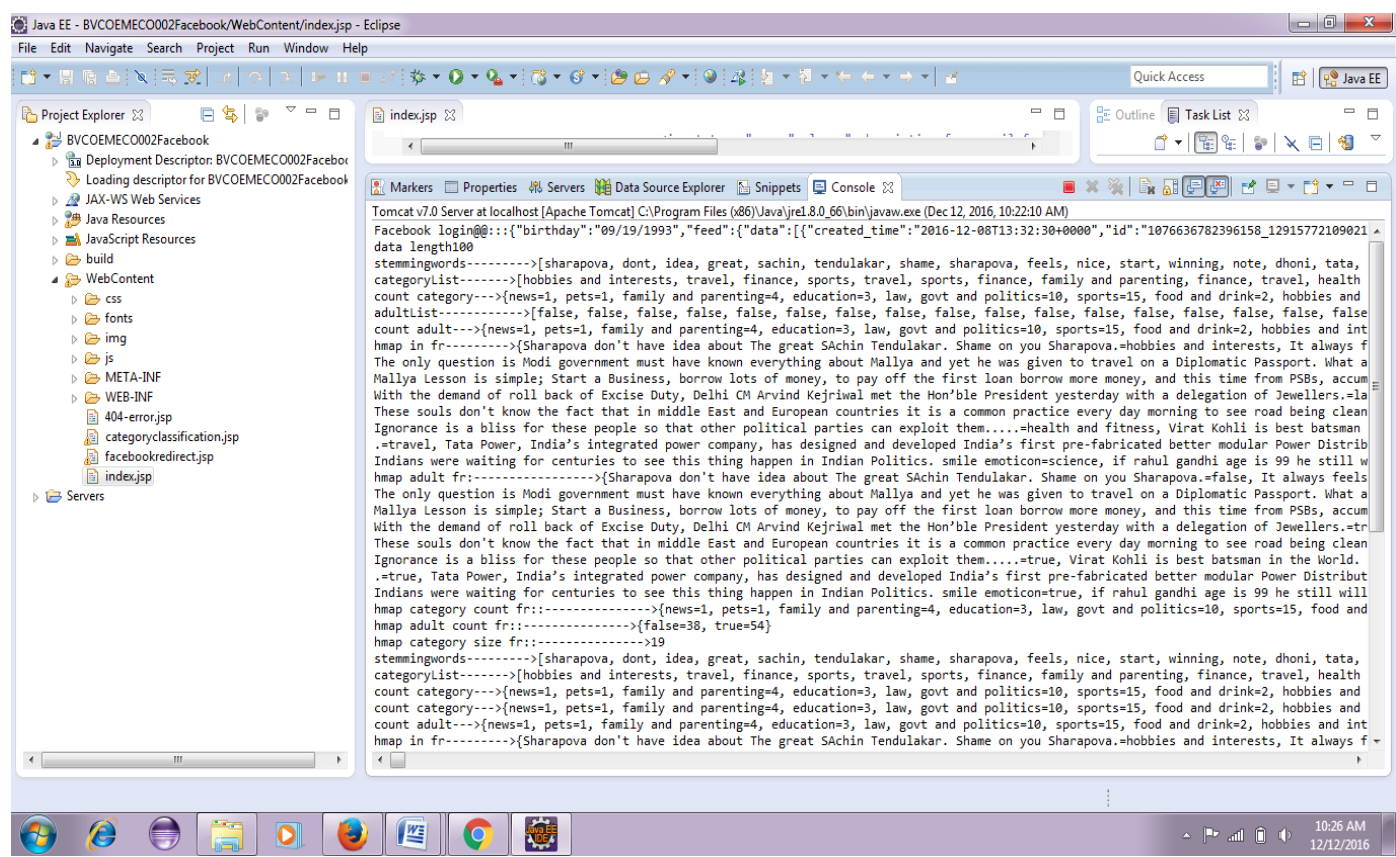

Figure 5. Classification and Categorization Result

Figure 6: Classification graph of facebook post.

Description: Figure (6) signify the graph for adult content and non adult content for facebook post at text and URL level. We have also represents the classification of facebook post into adult and non adult content. In Figure (6) X-axis represent the facebook post classification into adult and non adult and $\mathrm{Y}$ - axis represents the score of adult and non adult content.

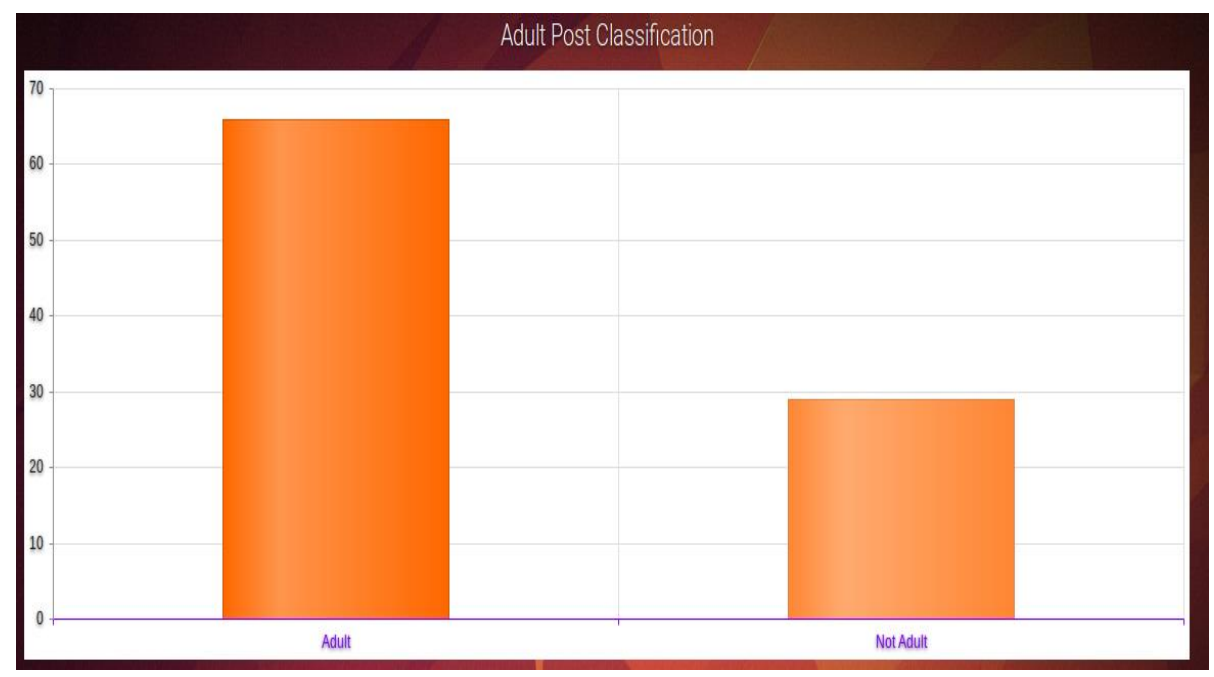

Figure 6. Result of Classification 
Figure 7: Categorization graph of post.

Description: We have also generate the graph for category of post about related topics such as business and industrial, law, government and politics, news, society, art and entertainment, sports, travel, education, style and fashion, science, careers, religion and spirituality, technology and computing, food and drink, health and fitness, pets, family and parenting, finance, hobbies and interests etc. In Figure (7) X-axis represents category of post which we have selected in our system. Y-axis represents score of category via facebook post.

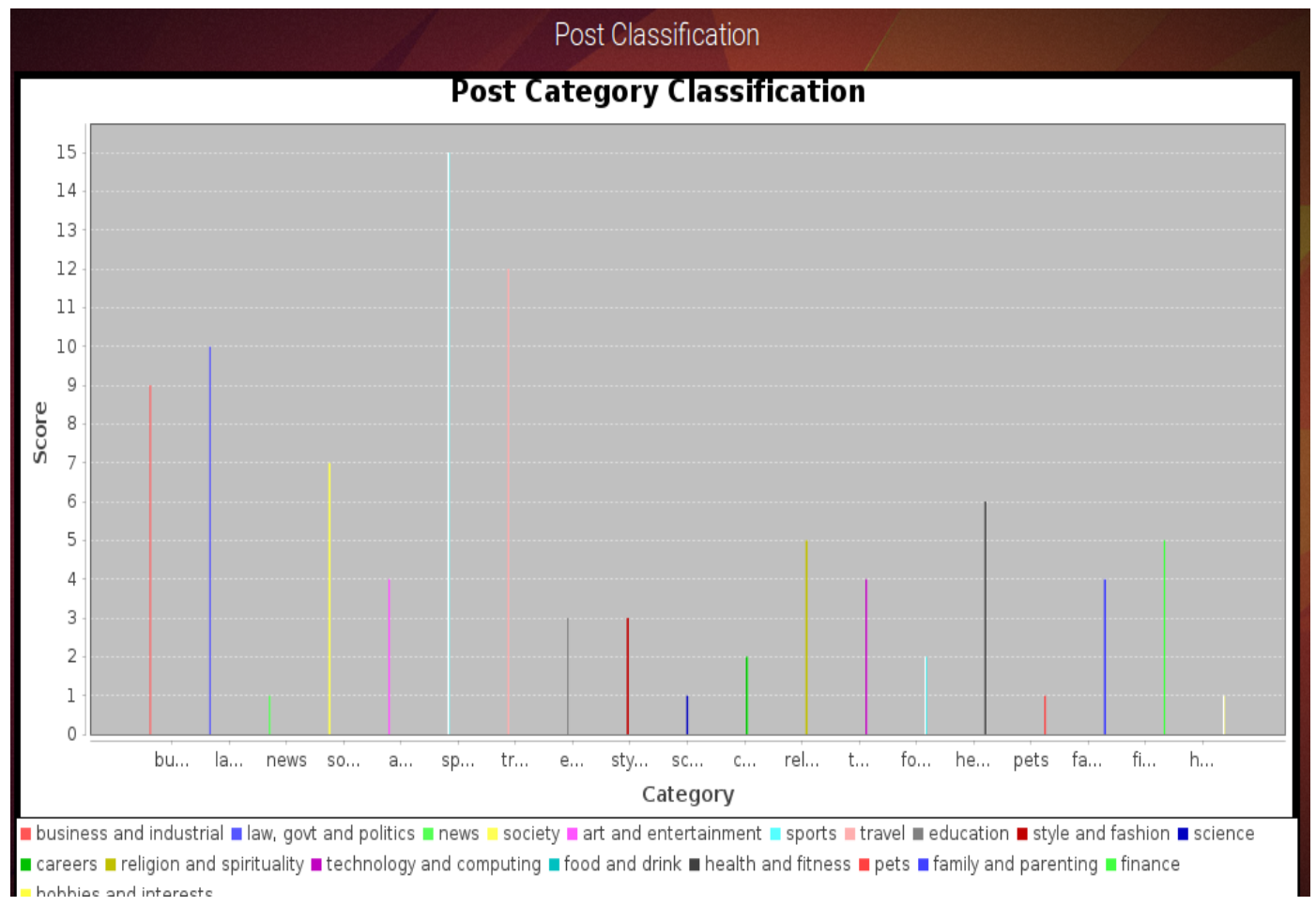

Figure 7. Result of Categorization

\section{Conclusion}

Here, we have propose system for facebook data classification using NLP, machine learning technique, our proposed methodology classify facebook post at text and URL level into adult and non adult classes. The main goal of proposed system is to address and solve adult content detection problem to provide possible solution to the facebook data classification, that goal we have achieved by Detecting, Classifying and categorizing from a post at text and URL level on facebook. In our proposed system we have performed research for text level (sentence-level post) and URL classification but in future this experiment can apply on different online social network sites. Our system is restricted within limited number of facebook post but in future one can apply this experiment on huge freely available data set of different online social sites.

\section{References}

[1] Hanqiang Cheng, Xinyu Xing, Xue Liu, and Qin Lv "ISC: An Iterative Social Based Classifier for Adult Account Detection on Twitter", IEEE Transactions on Knowledge and Data Engineering, Vol. 27, No. 4, pp. 1045-1056, April 2015.

[2] URLBlacklist[online]. http://urlblacklist.com.

[3] http://developers.facebook.com.

[4] H.-F. Yu, C.-H. Ho, Y.-C. Juan, and C.-J. Lin. "Libshorttext: A library for short-text classification and analysis[Online]", Available: http://www.csie.ntu.edu.tw/ cjlin/libshorttext. 
[5] Stein, T.; Chen, E.; and Mangla, K. "Facebook immune system. In Proceedings of the 4th Workshop on Social Network Systems", 8. ACM, 2011.

[6] Gao, H, Hu, J, Wilson, C, Li, Z, Chen, Y, and Zhao, B. Y. "Detecting and characterizing social spam campaigns", In Proceedings of the 10th ACM SIGCOMM conference on Internet measurement, page. 35-47, ACM, 2010.

[7] Gao Gao, H, Chen, Y, Lee, K, Palsetia, D, and Choudhary A. N. "Towards online spam filtering in social networks", In NDSS, 2012.

[8] Kurt Thomas, Chris Grier, Justin Ma, Vern Paxson, Dawn Song, Design and Evaluation of a Real-Time URL Spam Filtering Service :.

[9] Hongyu Gao et al, "Towards Online Spam Filtering”, International Journal of Innovative and Emerging Research in Engineering Volume 3, Special Issue 1, ICSTSD 2016.

[10] "Detecting Spam Classification on Twitter Using URL Analysis, Natural Language Processing and Machine Learning", International Journal of Innovative and Emerging Research in Engineering Volume 3, Special Issue 1, page. 141, ICSTSD 2016.

[11] Faraz Ahmed, Muhammad Abulaish, "An MCL-Based Approach for Spam Profile Detection in Online Social Networks", SMIEEE, 2012.

[12] T. Deselaers, L. Pimenidis, and H. Ney, "Bag-of-visual-words models for adult image classification and filtering", in Proc. IEEE Conf. Pattern Recognition, Dec. 2008, pp. 1-4.

[13] M. Fleck, D. Forsyth, and C. Bregler, "Finding naked people", in Proc. 10th Eur. Conf. Comput. Vis. pp. 593-602, 1996.

[14] M. Hammami, Y. Chahir, and L. Chen, "Webguard: A web filtering engine combining textual, structural, and visual content-based analysis", IEEE Trans. Knowl. Data Eng., vol. 18, no. 2, pp. 272284, Feb. 2006.

[15] M. Hepple, N. Ireson, P. Allegrini, S. Marchi, S. Montemagni, and J. G_omez, "Nlp-enhanced content filtering within the poesia project", in Proc. 4th Int. Conf. Lang. Resources Eval, p. 1, 2004.

[16] J. Ze Wang, J. Li, G. Wiederhold, and O. Firschein, "System for screening objectionable images", Computer Communication., vol. 21, no. 15, pp. 1355-1360,1998.

[17] H. Cheng, Y. Liang, X. Xing, X. Liu, R. Han, Q. Lv, and S. Mishra, "Efficient misbehaving user detection in online video chat services", in Proc. 5th ACM Int. Conf. Web Search Data Mining, pp. 2332, 2012.

[18] P. Kakumanu, S. Makrogiannis, and N. Bourbakis, "A survey of skin-color modeling and detection methods", Pattern Recognit., vol. 40, no. 3, pp. 1106-1122, 2007.

[19] X. Xing, Y.-1. Liang, H. Cheng, J. Dang, R. Han, X. Liu, Q. Lv, and S. Mishra, "Safevchat: Detecting obscene content and misbehaving users in online video chat services", in Proc. 20th Int. Conf. Companion World Wide Web, pp. 685-694, 2011.

[20] K9 web protection [Online]. Available: http://www.k9webprotection.com 
International Journal of Advanced Science and Technology

Vol.101 (2017) 